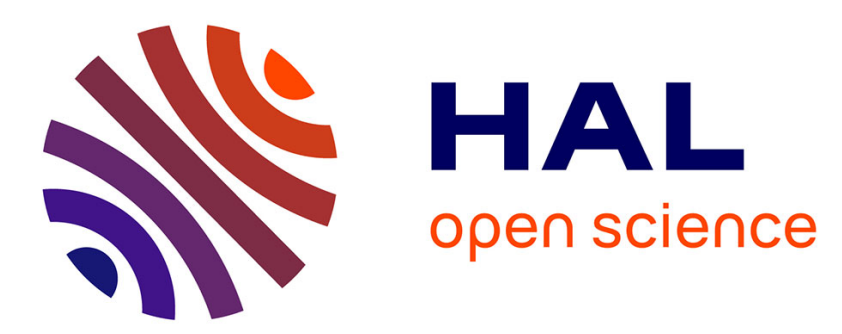

\title{
Pump-soliton nonlinear wave mixing in noise-driven fiber supercontinuum generation
}

\author{
M. Erkintalo, J.M. Dudley, G. Genty
}

\section{To cite this version:}

M. Erkintalo, J.M. Dudley, G. Genty. Pump-soliton nonlinear wave mixing in noise-driven fiber supercontinuum generation. Optics Letters, 2011, 36 (19), pp.3870-3872. 10.1364/OL.36.003870 . hal-00655040

\section{HAL Id: hal-00655040 \\ https://hal.science/hal-00655040}

Submitted on 14 Apr 2021

HAL is a multi-disciplinary open access archive for the deposit and dissemination of scientific research documents, whether they are published or not. The documents may come from teaching and research institutions in France or abroad, or from public or private research centers.
L'archive ouverte pluridisciplinaire HAL, est destinée au dépôt et à la diffusion de documents scientifiques de niveau recherche, publiés ou non, émanant des établissements d'enseignement et de recherche français ou étrangers, des laboratoires publics ou privés. 


\title{
Pump-soliton nonlinear wave mixing in noise-driven fiber supercontinuum generation
}

\author{
Miro Erkintalo, ${ }^{1, *}$ John M. Dudley, ${ }^{2}$ and Goëry Genty ${ }^{1}$ \\ ${ }^{1}$ Tampere University of Technology, Optics Laboratory, FI-33101 Tampere, Finland \\ ${ }^{2}$ Université de Franche-Comté, Institut FEMTO-ST, 25030 Besançon, France \\ *Corresponding author: miro.erkintalo@tut.fi
}

\begin{abstract}
We report on the experimental observation of nonlinear mixing between Raman-shifting solitons and residual pump radiation in supercontinuum generation in the long-pulse regime. This resonant coherent process results in the generation of strong and isolated spectral components on the long-wavelength normal dispersion regime of a fiber with two zero-dispersion wavelengths. Our observations are in excellent agreement with numerical simulations and calculated phase-matching conditions.
\end{abstract}

Soliton effects play a crucial role in the generation of broadband supercontinua (SC) in photonic crystal fibers (PCFs) [1,2]. Although soliton propagation is restricted to the anomalous dispersion regime, soliton dynamics can also cause spectral broadening into the high-frequency normal dispersion regime through a Cherenkov-like resonant radiation mechanism [3,4]. In a PCF with two zerodispersion wavelengths (ZDWs), the same mechanism can lead to the extension of the SC spectral bandwidth toward longer wavelengths $[5,6]$.

In addition to the direct generation of such phasematched radiation, of particular interest is also the case where a soliton mixes with a weak linear wave to generate new frequency components that are generally inaccessible through other spectral broadening processes. Such dynamics are governed by a Kerr-type nonlinear interaction of a strong soliton $(S)$ and a small-amplitude linear wave $(P)$ and can be described by processes of the form $\left|E_{S}\right|^{2} E_{P}$ or $E_{S}^{2} E_{P}^{*}$, where $E_{S}$ and $E_{P}$ represent the electric fields of the soliton and the linear wave, respectively, and the asterisk denotes the complex conjugate $[7,8]$. For convenience, in what follows, we refer to the $\left|E_{S}\right|^{2} E_{P}$ process as type I and the $E_{S}^{2} E_{P}^{*}$ process as type II. A particular feature of the type II process is that it depends coherently on the phases of the soliton and the small-amplitude wave [2]. Whereas the type I process has been experimentally observed in a directly seeded configuration [9] as well as in coherent [10] and incoherent SC generation [11], to our knowledge, the type II process has been observed only by coherent seeding of the soliton and the linear wave into orthogonal polarization modes [12].

In this Letter, we report on the experimental generation of spectral components of type II in the longwavelength normal dispersion regime of a PCF with two ZDWs. In our experiments, the nonlinear mixing process is not seeded but rather arises spontaneously from the nonlinear dynamics of noise-driven SC generation in the long-pulse regime. In contrast with previously identified dynamics [11], the physical mechanism responsible for these spectral components is associated with the temporal overlap between ultrashort redshifting solitons generated through initial modulation instability and the extended trailing edge of the residual pump pulse.
As a result, these spectral components have a large amplitude and are significantly isolated by nearly $200 \mathrm{~nm}$ from the SC spectrum.

Our experimental setup is shown in Fig. 1. We use a mode-locked Ti:sapphire laser operating at a repetition rate of $80 \mathrm{MHz}$ that produces $3.5 \mathrm{ps}$ pulses (FWHM) with $310 \mathrm{~W}$ peak power. The pulses are launched into an $86 \mathrm{~cm}$ long PCF (NKT Photonics NL-PM-750) with two ZDWs at 760 and $1260 \mathrm{~nm}$. The center wavelength of the pump pulses is tuned to $960 \mathrm{~nm}$, which lies in the anomalous dispersion region of the PCF. A half-wave plate and a polarizer are used to adjust the polarization of the input pulses along one of the principal axes of the polarization maintaining fiber. In our experiments, we carefully checked that all the SC spectral components were generated along the same axis.

In the long-pulse regime, SC dynamics are highly sensitive to the input noise with large shot-to-shot amplitude and phase fluctuations [13]. This noise sensitivity is reflected in the smoothness of the measured average output spectrum shown in Fig. 2(a). The SC spectrum extends predominantly into the long-wavelength side since the pump wavelength is located far from the first ZDW. Interestingly, however, in contrast with previously reported results, the spectrum beyond the second ZDW exhibits distinct features: a broad peak centered around $1400 \mathrm{~nm}(A)$ and a much narrower peak at $1600 \mathrm{~nm}(B)$
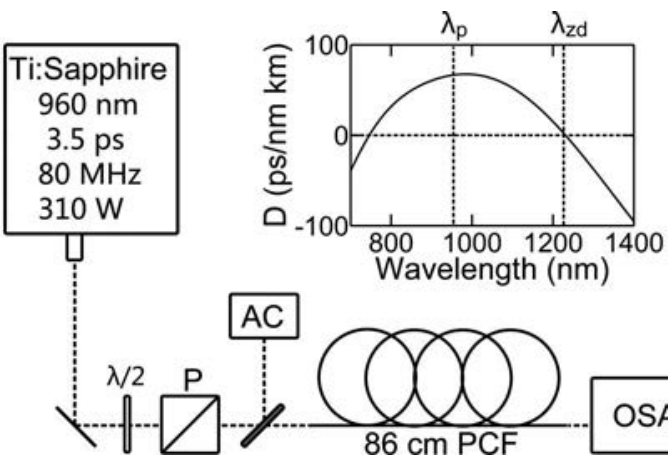

Fig. 1. Experimental setup and fiber dispersion profile. $\lambda / 2$, half-wave plate; $P$, polarizer; AC, autocorrelator; OSA, optical spectrum analyzer; $\lambda_{P}$, pump wavelength; $\lambda_{\mathrm{zd}}$, long-wavelength ZDW. 
separated by a plateau $(C)$. This contrasts with the single broad Cherenkov peak that is typically observed for SC generation in PCFs with two ZDWs [5,6,11].

In order to gain more insight, we have numerically generated an ensemble of 1000 simulations using the generalized nonlinear Schrödinger equation with a stochastic quantum noise term [1]. Our simulations use the experimental input pulse parameters and we assume a value of $\gamma=0.1 \mathrm{~W}^{-1} \mathrm{~m}^{-1}$ for the nonlinear coefficient of the PCF. The dispersion profile of the PCF (shown in Fig. 1) was obtained by fitting an eighth-order polynomial onto a curve provided by the retailer. The mean spectrum obtained from the simulations is plotted in Fig. 2(b) and shows excellent qualitative and quantitative agreement with the experimental spectrum. In particular, the twopeak spectral structure separated by a plateau beyond the long-wavelength ZDW can be seen to be very well reproduced by the simulations.

In order to clarify the origin of the double peak structure, it is instructive to consider one particular realization of the ensemble. To this end, Fig. 3 illustrates the spectrogram representation of a selected simulation at two propagation distances of 76 and $86 \mathrm{~cm}$, where both the spectral peaks $(A)$ and $(B)$ are clearly observed. The figure caption provides a link to a movie (Media 1) illustrating the dynamical spectrogram evolution with distance. Several observations can be made. First, we can see how a large number of short solitons have emerged near the center of the pump pulse envelope. These solitons experience the soliton self-frequency shift (SSFS) and are delayed with respect to the remains of the pulse envelope along propagation. As the solitons spectrally approach the long-wavelength ZDW, the SSFS stabilizes with the emission of a dispersive wave into the normal dispersion regime [5]. Comparison with Fig. 2 clearly reveals that it is precisely this mechanism that can be associated with the broad spectral component $(A)$. On the other hand, the narrowband component $(B)$ can also be observed at around $1600 \mathrm{~nm}$ in Fig. 3, and it is clear that the generation process associated with this spectral component follows the time-domain motion of the soliton. Simulations over a longer propagation distance (see Media 1) show that the efficiency of the generation of this component decreases as the soliton loses its temporal overlap with the pump pulse residue. This suggests that the narrowband component $(B)$ arises from a soliton-dispersive wave resonance of either type I or type II, where the remains of the pump envelope acts as the linear wave of the interaction. (a) Experiment

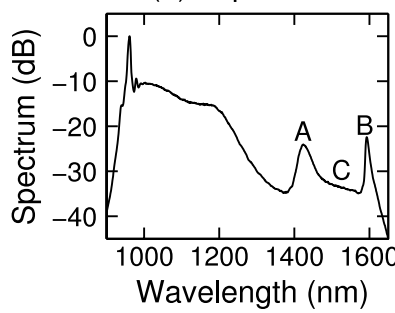

(b) Simulation

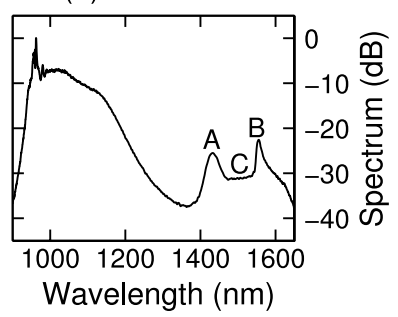

Fig. 2. (a) Measured and (b) simulated average SC spectra generated by $3.5 \mathrm{ps}$ pulses at $960 \mathrm{~nm}$ in an $86 \mathrm{~cm}$ long PCF. The labels $A, B$, and $C$ refer to different wave-mixing processes, as discussed in the text.

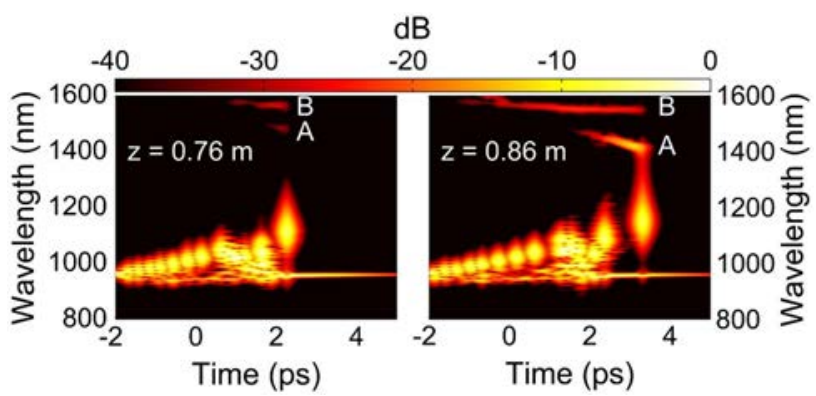

Fig. 3. (Color online) Time-frequency illustration of one particular simulation at two different propagation lengths displaying two distinct dispersive wave peaks. The spectrograms were calculated using a $200 \mathrm{fs}$ gate function. Media 1 is a movie of the evolution over a longer propagation distance.

In order to confirm that the nonlinear superposition of the trailing edge residue of the pump with a Ramanshifting soliton is indeed the mechanism that seeds the generation of the isolated narrowband spectral component, we calculate the phase-matching condition corresponding to type II nonlinear superposition as [7,8]:

$$
\beta(\omega)=-\beta\left(\omega_{P}\right)+\beta_{S}(\omega)+\beta_{S}\left(\omega_{P}\right),
$$

where $\beta(\omega)$ is the frequency-dependent propagation constant of the fiber, $\beta_{S}(\omega)=\beta\left(\omega_{S}\right)+\beta_{1}^{(S)}\left(\omega-\omega_{S}\right)+\gamma P_{S} / 2$, and $\beta_{1}^{(S)}=d \beta /\left.d \omega\right|_{\omega=\omega_{S}}$. Here, $\omega_{P}$ and $\omega_{S}$ represent the frequencies of the linear pump residue and soliton, respectively, and $P_{S}$ is the soliton peak power. Extracting the peak power and center wavelength of the Raman-shifting soliton from the numerical simulation shown in Fig. 3, we calculate the theoretical phase-matched wavelength for the process described by Eq. (1). The frequency associated with the trailing edge of the input pulse is $\omega_{P}=2 \pi c / \lambda_{P}$, where $\lambda_{P}$ is the initial pump wavelength. Figure 4 compares the predicted phase-matched wavelength as a function of propagation distance with the wavelength of the isolated narrowband spectral component as directly extracted from the simulations. For completeness, we also plot the wavelength of the broadband Cherenkov-type peak $(A)$ and compare it with the theoretically calculated resonance wavelength using the well-known condition $\beta(\omega)=\beta_{S}(\omega)$. We further emphasize that the type I phase-matching condition does not match any of our observations.

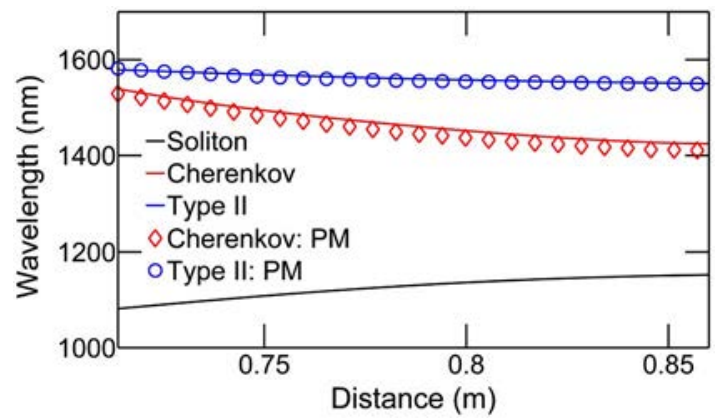

Fig. 4. (Color online) Wavelengths extracted from simulations (solid curve) and calculated from phase-matching (PM) conditions (diamonds and circles). Black, soliton; red, Cherenkov; blue, type II wave mixing. 
There is an excellent agreement between the theoretically calculated phase-matched wavelengths and the wavelengths extracted from the numerical simulations. This confirms that (i) the broadband spectral component (A) results from the continuous emission of the Ramanshifting soliton as it approaches the second ZDW, and (ii) that the narrowband spectral feature $(B)$ is a manifestation of the type II phase-matched soliton-pump residue nonlinear interaction. Significantly, we also note that the type II phase-matching condition is much less sensitive to changes in the Raman-shifting soliton wavelength as compared to that governing the direct emission of Cherenkov radiation. This explains both the relatively narrow bandwidth and large amplitude characteristics of the isolated long-wavelength component $(B)$ as observed both experimentally and numerically.

In addition, careful inspection of the simulation shown in Fig. 3 shows very little energy content between the broadband and narrowband dispersive wave components while a clear plateau (labeled $C$ in Fig. 2) is observed in both the measured and numerically simulated average spectra. In fact, examination of each individual simulation reveals that the plateau region $(C)$ results from dispersive waves generated by random soliton collisions that occur during the initial turbulent phase of soliton formation [14,15]. The random character of the collisions results in a widely distributed wavelength range of the dispersive waves, hence explaining the plateaulike characteristics. Furthermore, while the resonant peaks ( $A$ and $B$ ) can be observed in most of the realizations, only approximately $10 \%$ of the observed events display collision dynamics that contribute significantly to the plateau region $(C)$, resulting in a reduced average energy density in the plateau as compared to that in the spectral peaks ( $A$ and $B$ ).

In conclusion, we have demonstrated experimentally and numerically the generation of an isolated narrowband spectral component separated by nearly $200 \mathrm{~nm}$ from the SC spectrum. This spectral component was shown to arise from a phase-matched wave-mixing interaction between a soliton and the remains of the pump trailing edge in long-pulse SC generation in a fiber with two ZDWs. The dynamics are self-seeded by Ramanshifting solitons that temporally overlap with the residual pump pulse along propagation. We expect this mechanism to provide an additional degree of freedom in tailoring SC spectra in fibers with complex dispersion profiles. From an application perspective, the pumpsoliton dynamics reported here could allow for the generation of long-wavelength components particularly useful for accessing spectroscopy in novel regimes.

We thank the Academy of Finland (research grants 121953, 130099, and 132279), the Institut Universitaire de France, the French Agence Nationale de la Recherche project IMFINI (ANR-09-BLAN-0065), and the graduate school of Tampere University of Technology.

\section{References}

1. J. M. Dudley, G. Genty, and S. Coen, Rev. Mod. Phys. 78, 1135 (2006).

2. D. V. Skryabin and A. V. Gorbach, Rev. Mod. Phys. 82, 1287 (2010).

3. P. K. A. Wai, C. R. Menyuk, Y. C. Lee, and H. H. Chen, Opt. Lett. 11, 464 (1986).

4. N. Akhmediev and M. Karlsson, Phys. Rev. A 51, 2602 (1995).

5. D. V. Skryabin, F. Luan, J. C. Knight, and P. St. J. Russell, Science 301, 1705 (2003).

6. G. Genty, M. Lehtonen, H. Ludvigsen, and M. Kaivola, Opt. Express 12, 3471 (2004).

7. A. V. Yulin, D. V. Skryabin, and P. St. J. Russell, Opt. Lett. 29, 2411 (2004).

8. D. V. Skryabin and A. V. Yulin, Phys. Rev. E 72, 016619 (2005).

9. A. Efimov, A. V. Yulin, D. V. Skryabin, J. C. Knight, N. Joly, F. G. Omenetto, A. J. Taylor, and P. St. J. Russell, Phys. Rev. Lett. 95, 213902 (2005).

10. A. Efimov, A. Taylor, F. Omenetto, A. Yulin, N. Joly, F. Biancalana, D. Skryabin, J. C. Knight, and P. St. J. Russell, Opt. Express 12, 6498 (2004).

11. B. H. Chapman, J. C. Travers, S. V. Popov, A. Mussot, and A. Kudlinski, Opt. Express 18, 24729 (2010).

12. A. Efimov, A. J. Taylor, A. V. Yulin, D. V. Skryabin, and J. C. Knight, Opt. Lett. 31, 1624 (2006).

13. J. M. Dudley, G. Genty, F. Dias, B. Kibler, and N. Akhmediev, Opt. Express 17, 21497 (2009).

14. M. Erkintalo, G. Genty, and J. M. Dudley, Opt. Lett. 35, 658 (2010).

15. M. Erkintalo, G. Genty, and J. M. Dudley, Opt. Express 18, 13379 (2010). 